\title{
A MODEL FOR THE OPTICAL PROPERTIES OF POROUS GRAINS
}

\author{
J. I. Hage AND J. Mayo Greenberg \\ University of Leiden \\ Received 1989 November 13; accepted 1990 March 20
}

\begin{abstract}
A numerical method, based on an integral representation of Maxwell's equations, is used to calculate scattering properties of model porous aggregates of small particles. It is shown that increasing porosity can lead to enhanced absorption and emission of radiation and a lower albedo. One application is to show how strongly the $9.7 \mu \mathrm{m}$ emission feature of a piece of "astronomical silicate" matter depends on its porosity. The integral method is compared with the well-known coupled dipole approach for the case of a solid sphere. The validity of the Maxwell-Garnett effective medium theory is shown for certain cases where the individual particles in the aggregate satisfy the Rayleigh size conditions.
\end{abstract}

Subject headings: comets - infrared: spectra - interstellar: grains - polarization - radiative transfer

\section{INTRODUCTION}

There are indications that a large number of porous, or "fluffy," particles exist in the solar system. These appear as interplanetary dust (Giese et al. 1978; Greenberg 1980), and in the comae of comets (Greenberg and Hage 1990). Micrometeorites collected in the atmosphere also have a fluffy structure (Brownlee, Tomandl, and Hodge 1976), and meteors are known to have a density too low to be that of solid particles (Verniani 1973). Important clues concerning these porous particles can be gained from, among other things, the interpretation of their thermal emission. The thermal emission of a particle at a temperature $T$ is given by

$$
F(\lambda, T) \sim C_{\text {abs }}(\lambda) B(\lambda, T),
$$

where $\lambda$ is the wavelength, $C_{\text {abs }}$ the absorption cross section of the particle, and $B(\lambda, T)$ the Planck function. The wavelength dependence of the absorption cross section determines whether the particle will produce an emission feature. This quantity depends on the size, shape, and composition of the grain, so that modeling of thermal spectra and subsequent fitting of observations can give information concerning these characteristics. An interesting and important effect, which is considered in this paper, is that a particle as a whole may not exhibit an emission feature even though the material of which it is made does have a characteristic absorption profile. This was shown analytically by van de Hulst (1957) for an absorbing particle with a refractive index $m$ which satisfies $|m-1| \ll 1$. When such a particle becomes very large compared with the wavelength, it behaves like a blackbody and its efficiency for absorption, $Q_{\text {abs }}$ (defined as $C_{\text {abs }} / G$, where $G$ is a mean projected particle area), is then equal to unity, independent of the wavelength. This effect also occurs for absorbing particles for which $|m-1|$ is larger. For example, Figure 1 shows that $Q_{\text {abs }}$ for spheres with refractive indices $m=1.33-0.25 i$ and $m=1.33-0.50 i$, converges to a value close to unity as the size parameter $x$ increases ( $x=2 \pi a / \lambda$, where $a$ is the sphere radius). For convenience we shall use the term "saturation of the absorption" from here on to mean that the absorption cross section of a particle is not changed significantly by an increase in its material absorptivity (i.e., and increase in $|\operatorname{Im} m|)$. The saturation effect is described by Greenberg and Hage (1990) for the case of silicate spheres and applied to the interpretation of the thermal spectrum of comet Halley. Using the fact that porous particles saturate at larger sizes compared with equally massive solid particles, Greenberg and Hage postulate the existence of a large amount of porous dust in the coma of the comet in order to explain the spectral emission at 3.4 and $9.7 \mu \mathrm{m}$.

Because of the widespread existence of porous particles in the solar system there is a need to have a feeling for the optical properties of such particles. A key question, concerning, e.g., the quantitative interpretation of thermal spectra of porous particles, is, How does $C_{\text {abs }}$ of porous particles behave as a function of size, refractive index of the component parts, and porosity? Or, put in another way, to what extent is the scattering by a fluffy aggregate of small particles intermediate between the behavior of a large, solid particle and the properties of a cloud of the constituent small particles? Another interesting problem, in relation to the scattering of sunlight, is to determine the behavior of the albedo of such fluffy structures. The answers require electrodynamical scattering calculations for inhomogeneous scatterers. Calculations to obtain the extinction by aggregates of small particles have been described by Wright (1987), whose method is approximate and limited to aggregates which are smaller than the wavelength. Jones (1988) used very simple shapes (core-mantle spheres) and MaxwellGarnett's effective medium theory (Maxwell-Garnett 1904) to approximate extinctions of porous particles. In his paper Jones stressed the need for an exact method to describe the scattering by porous particles.

The present paper reports results of calculations which illustrate the behavior of some optical properties of a special kind of porous aggregate as a function of porosity, size, and refractive index. In particular, attention is paid to porous aggregates such as are thought to exist in the coma of comet Halley. These calculations are an application of an approximate numerical implementation of the exact representation of the macroscopic Maxwell's equations. The well-known coupled dipole method by Purcell and Pennypacker (1973) is a different approach to the solution of the electromagnetic scattering problem, which can also be derived from the integral equation, as shown in the Appendix to this paper. As an application we show how the $9.7 \mu \mathrm{m}$ silicate emission band of comet Halley depends on the porosity of the emitting dust. 


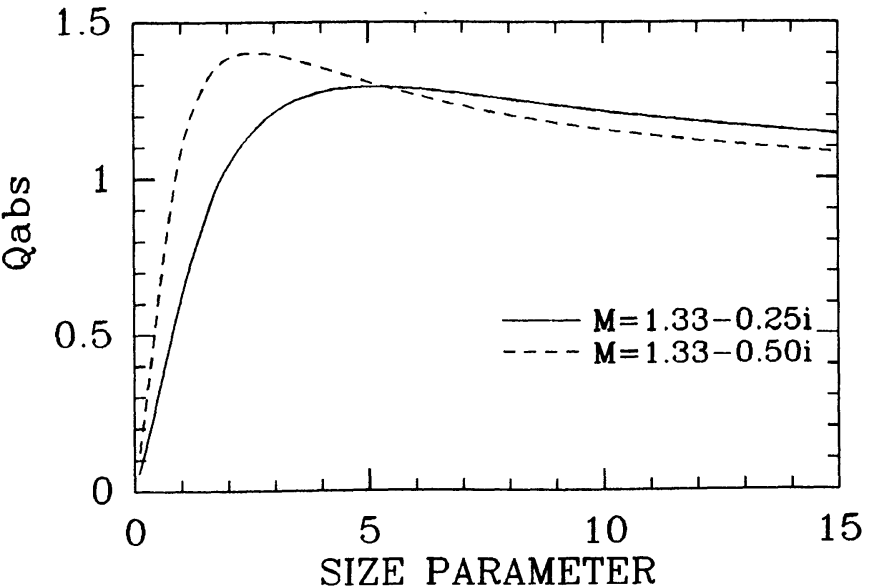

FIG. 1.-Efficiency for absorption for spheres, for two refractive indices, as a function of the size parameter.

\section{A MODEL FOR FLUFFY AGGREGATES}

In this section we derive the model of a porous aggregate which we have used in our calculations. This model should primarily be able to represent a fluffy dust particle such as could exist in the coma of the comet, but it may also represent various other kinds of porous particles. As a basis, we will use the model of comets proposed by Greenberg (Greenberg 1982, 1986), in which a comet is pictured as an enormous aggregate of interstellar dust particles with icy mantles. Studies of the interstellar dust (Greenberg 1985) indicate that the individual dust particles have elongated cores of an amorphous silicate, about $0.1 \mu \mathrm{m}$ thick and roughly 3 times as long. It is inferred that around this core a mantle of "organic refractory" material exists which makes the whole grain about $1 \mu \mathrm{m}$ long and $0.3 \mu \mathrm{m}$ thick (see Fig. $2 a$ ). In the denser molecular clouds an additional mantle of mostly water ice condenses on the dust particles. The ultimate result which is used to represent a mean precometary dust grain is shown in Figure $2 b$. A consequence of the model is that when a comet comes close to the Sun, interstellar dust aggregates of various sizes are blown off its
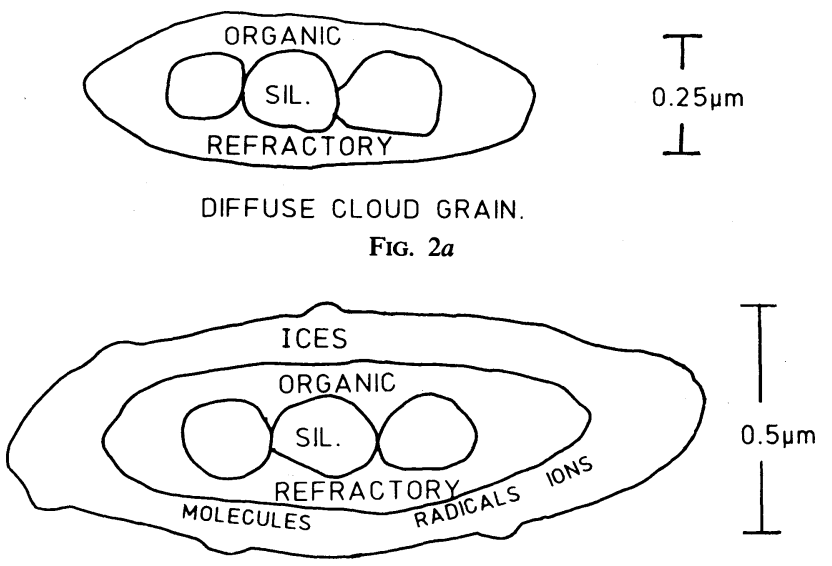

PRECOMETARY GRAIN

FIG. $2 b$

FIG. 2.-(a) Schematic representation of a typical interstellar dust grain. (Adapted from Greenberg, Zhao, and Hage 1989.) (b) The same interstellar grain as it would appear inside a molecular cloud. surface, and from these the icy mantles evaporate. The aggregates that are left over, which fill a coma, are loosely packed aggregates consisting of the particles shown in Figure $2 a$. These aggregates must be responsible for the emission observed from the coma of comet Halley, and so it is for these fluffy structures that we want to perform representative scattering calculations.

For the purpose of light-scattering calculations a truly general coma dust particle as pictured above must be described by a very large number of parameters. These include the size and shape of the aggregate; the degree of porosity, the sizes, shapes, positions, and orientations of the subparticles; and the complex refractive index of the materials in the aggregate. In order to limit the amount of computation to manageable proportions, it proved necessary to reduce the number of variables. To accomplish this, we have chosen to model the cometary dust aggregates in the following way. We consider such a fluffy particle to be a collection of identical homogeneous cubical subvolumes mounted in a cubic lattice within a cubic volume. All the cubical subvolumes have the same refractive index and orientation. Porosity is simulated by randomly choosing the sites of the filled subvolumes within the cubic lattice. This cubic lattice is defined to be the bounding volume of the aggregate. Examples of such a model particle are shown in Figure 3. We define a porosity parameter, $P$, as the fractional amount of vacuum within the enclosing volume. The size parameter, $X$, of the aggregate is taken as $2 \pi a_{\text {eq }} / \lambda$, where $a_{\text {eq }}$ equals the radius of the sphere having the same volume as the bounding volume of the aggregate. The size parameter of the subvolumes, $x$, is defined in the same way. We have performed calculations for cubic lattices containing from $10^{3}$ to $54^{3}$ lattice sites. The wavenumber, $k=2 \pi / \lambda$, was taken to be
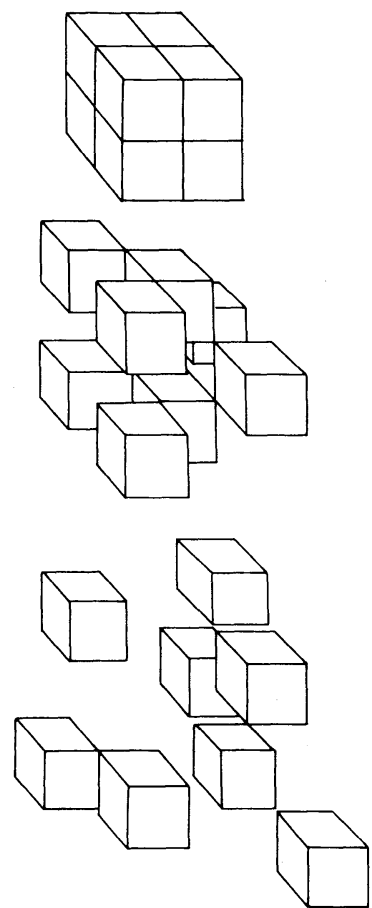

Fig. 3.-Models for porous aggregates. Shown are assemblies of 8 cubic subvolumes. Top: 8 subvolumes in a $2 \times 2 \times 2$ lattice, representing a solid aggregate. Middle: 8 subvolumes in a $3 \times 3 \times 3$ lattice, $P=0.7$. Bottom: 8 subvolumes in a $4 \times 4 \times 4$ lattice, $P=0.875$. 
0.3 , in units where the length of the lattice spacing (or, equivalently, the size of the subvolumes) equals unity. This corresponds to the subvolumes having a fixed size parameter of about 0.19 and the aggregates having size parameters between approximately 1.9 and 10 , which is representative of the size parameters of the coma dust particles with radii between 3 and $16 \mu \mathrm{m}$ for the silicate emission feature which is at $9.7 \mu \mathrm{m}$. In our calculations, we have mostly used an orientation of the cubic lattice such that the incoming plane wave is directed perpendicularly to a cube face and is polarized parallel to another face. This restriction has no effect on our basic conclusion. The remaining variables describing a model aggregate are thus the size parameter, the porosity, which can range from 0 to 1 , and the complex refractive index of the individual particles.

\section{THEORY}

In our notation we will use the same conventions as van de Hulst (1957), i.e., Gaussian units and a time factor $\exp (i \omega t), \omega$ being the angular frequency. Note that this choice results in a refractive index with a negative imaginary part. The magnetic permeability is taken as $\mu=1$. The particle material is then completely characterized by a complex refractive index $m^{2}=\epsilon-4 \pi i \sigma / k c$, where $\epsilon$ is the dielectric constant, $\sigma$ is the conductivity, and $k=2 \pi / \lambda ; \lambda$ is the wavelength of the radiation in vacuum and $c$ the speed of light. The vacuum regions inside and outside of the particle have $\mu=1, m=1$, and $\sigma=0$. The incident field is a monochromatic plane wave. Generally, we will use

$$
E^{\text {in }}(\boldsymbol{r})=\hat{\boldsymbol{x}} e^{-i k z+i \omega t},
$$

i.e., a plane wave of unit intensity traveling in the positive $z$-direction and polarized in the positive $x$-direction. The size of the particle must be finite, but the shape in not constrained. If one writes

$$
E(r)=E_{1}(r)+E^{\text {in }}(r),
$$

where $\boldsymbol{E}(\boldsymbol{r})$ is the total electric field at the point $\boldsymbol{r}, \boldsymbol{E}^{\mathrm{in}}(\boldsymbol{r})$ the incident plane wave, and $E_{1}(r)$ the scattered radiation, the macroscopic Maxwell's equations become

$$
\nabla \cdot E(r)=0
$$

and

$$
\nabla^{2} E_{1}(\boldsymbol{r})+k^{2} E_{1}(\boldsymbol{r})=-k^{2}\left[m^{2}(\boldsymbol{r})-1\right] \boldsymbol{E}(\boldsymbol{r}) .
$$

Equation (5) has the following solution which also satisfies the boundary conditions (see, for example, Yaghjian 1980 and Morse and Feshbach 1953):

$$
\boldsymbol{E}\left(\boldsymbol{r}_{1}\right)=\boldsymbol{E}^{\mathrm{in}}\left(\boldsymbol{r}_{1}\right)+\frac{k^{2}}{4 \pi} \iiint_{V}\left[m^{2}\left(\boldsymbol{r}_{2}\right)-1\right] \boldsymbol{E}\left(\boldsymbol{r}_{2}\right) \mathbf{G}\left(\boldsymbol{r}_{1}, \boldsymbol{r}_{2}\right) d^{3} \boldsymbol{r}_{2},
$$

where $\mathbf{G}$ is the divergence-free Green's dyadic for the vector Helmholtz equation, $\boldsymbol{r}_{1}$ is the point of observation, and $\boldsymbol{r}_{2}$ is a point inside the particle. The integration extends over the volume of the particle. The physical interpretation of equation (6) is that every point in the particle which is not vacuum radiates like a dipole whose magnitude and phase are determined by the local electric field and local refractive index. In full, equation (6) reads

$$
\begin{aligned}
\boldsymbol{E}\left(\boldsymbol{r}_{1}\right)= & \boldsymbol{E}^{\mathrm{in}}\left(\boldsymbol{r}_{1}\right)-\frac{m^{2}\left(\boldsymbol{r}_{1}\right)-1}{3} \boldsymbol{E}\left(\boldsymbol{r}_{1}\right) \\
& +\frac{k^{2}}{4 \pi} \iiint_{v}\left[m^{2}\left(\boldsymbol{r}_{2}\right)-1\right]\left\{\boldsymbol{E}\left(\boldsymbol{r}_{2}\right)\left(1+\frac{1}{i k R}-\frac{1}{k^{2} R^{2}}\right)\right. \\
& \left.-\hat{\boldsymbol{R}}\left[\hat{\boldsymbol{R}} \cdot \boldsymbol{E}\left(r_{2}\right)\right]\left(1+\frac{3}{i k R}-\frac{3}{k^{2} R^{2}}\right)\right\} \\
& \times \frac{\exp (-i k R)}{R} d^{3} \boldsymbol{r}_{2},
\end{aligned}
$$

where $R=\left|r_{1}-r_{2}\right|$ and $\hat{R}=\left(r_{1}-r_{2}\right) / R$. The principal value of the integral must be taken. Equation (7) is the basic equation which has to be solved for the vector field $\boldsymbol{E}(\boldsymbol{r})$ inside the particle using numerical methods. It is important to note that because of our choice of conventions, our equation (7) may contain sign differences compared with the corresponding expression presented by other workers. Equation (7) is exact and can be applied straightforwardly to porous particles, since the particle structure is contained in $m(\boldsymbol{r})$. For homogeneous particles the coupled dipole method follows from equation (7) by making a few simplifying approximations as shown in the Appendix to this paper.

By integrating the Poynting vector, one finds that the cross sections for absorption and extinction in terms of the electric field in the particle are, for an incident wave of unit intensity (Jackson 1975 or see, e.g., Greenberg 1960 for the scalar wave quantum mechanical analog),

$$
C_{\text {abs }}=-k \iiint_{v} \operatorname{Im}\left[m^{2}(r)\right]|E(r)|^{2} d^{3} r
$$

and

$$
C_{\text {ext }}=-k \operatorname{Im}\left\{\iiint_{v}\left[m^{2}(\boldsymbol{r})-1\right] \boldsymbol{E}(\boldsymbol{r}) \cdot\left[\boldsymbol{E}^{\mathrm{in}}(\boldsymbol{r})\right]^{*} d^{3} \boldsymbol{r}\right\} .
$$

The scattered amplitude is obtained by taking the limit $\left|\boldsymbol{r}_{1}\right| \gg$ $\left|\boldsymbol{r}_{2}\right|$ in equation (7):

$$
\begin{aligned}
\boldsymbol{E}^{\text {scat }}\left(\boldsymbol{r}_{1}\right)= & \frac{k^{2}}{4 \pi} \frac{\exp \left(-i k\left|\boldsymbol{r}_{1}\right|\right)}{\left|\boldsymbol{r}_{1}\right|} \\
& \times \iiint_{v}\left[m^{2}\left(\boldsymbol{r}_{2}\right)-1\right]\left\{\boldsymbol{E}\left(\boldsymbol{r}_{2}\right)-\hat{\boldsymbol{r}}_{1}\left[\hat{\boldsymbol{r}}_{1} \cdot \boldsymbol{E}\left(\boldsymbol{r}_{2}\right)\right]\right\} \\
& \times \exp \left(-i k \boldsymbol{r}_{2} \cdot \hat{\boldsymbol{r}}_{1}\right) d^{3} \boldsymbol{r}_{2},
\end{aligned}
$$

where $\hat{\boldsymbol{r}}_{1}=\boldsymbol{r}_{1} /\left|\boldsymbol{r}_{1}\right|$. The scattering amplitudes $S_{i}$, as defined by van de Hulst (1957), follow from equation (10). Last, we define the albedo as

$$
\text { Albedo }=\frac{C_{\mathrm{ext}}-C_{\mathrm{abs}}}{C_{\mathrm{ext}}} .
$$

\section{NUMERICAL PROCEDURE}

We have solved equation (7) numerically to find the vector field $\boldsymbol{E}(\boldsymbol{r})$ inside the particle for various situations, using the method of moments (Harrington 1968). The method of moments, as used here, involves the choice of a discretization scheme to convert the linear integral equation (7) into a set of simultaneous linear equations, represented by the matrix equation $\mathbf{A y}=\boldsymbol{b}$. Here $\mathbf{A}$ is a known matrix, $\boldsymbol{b}$ a known vector, and 
$y$ an unknown vector. For simplicity we assume that the electric field is constant inside the cubical subvolumes. Since these cubical subvolumes have a finite size, we note that a higher order expansion will probably lead to more accurate results, but at the cost of increasing the number of equations. The rest of the integrand in equation (7), describing the interaction between the subvolumes and defining the matrix elements of $\mathbf{A}$, is evaluated at the centers of these subvolumes. An exception is made for the matrix elements which correspond to the interaction of a subvolume with itself. In that case the cubical subvolume is approximated by an equal-volume sphere, and the integrand is determined analytically. The net result is that equation (7) is approximately represented by the matrix equation

$$
\mathbf{A} E_{v}=E_{v}^{\text {in }} .
$$

Here $E_{v}$ and $E_{v}^{\text {in }}$ are $6 N_{c}$-dimensional vectors which contain the values of the total electric field and the incident field in the subvolumes throughout the particle, where $N_{c}$ is the number of subvolumes. Equation (12) must be solved for $\boldsymbol{E}_{\boldsymbol{v}}$. For a full description of the method of moments applied to scattering by a particle in this way, we refer to Livesay and Chen (1974).

The matrix $\mathbf{A}$ is not necessarily positive definite. To solve equation (12) for $\boldsymbol{E}_{v}$, we have used the FORTRAN routine SPARSE as given by Press et al. (1986), which implements the interative conjugate gradient method. Our choice for the conjugate scheme to solve equation (12) was inspired by its proven applicability to similar problems in the past (see, e.g., van den Berg 1984; Peterson and Mittra 1985). The routine SPARSE solves the system $\mathbf{A} \boldsymbol{E}_{v}-\boldsymbol{E}_{v}^{\text {in }}=0$ numerically to a prescribed accuracy $\epsilon$, by iteratively finding the minimum of the functional $\left|\mathbf{A} \boldsymbol{E}_{v}-\boldsymbol{E}_{v}^{\text {in }}\right|^{2}$. This procedure does not require the matrix $\mathbf{A}$ to be positive definite.

The number of iterations required is determined by the accuracy of the initial guess for $\boldsymbol{E}_{v}$ and by an accuracy criterion $\epsilon$, which is to be suitably chosen. As a starting vector we have always taken $\boldsymbol{E}_{v}=\left[3 /\left(m^{2}+2\right)\right] \boldsymbol{E}_{v}^{\text {in }}$. The iteration ends when the quantity $\left|\mathbf{A} \boldsymbol{E}_{v}-\boldsymbol{E}_{v}^{\text {in }}\right|^{2}$ becomes smaller than $6\left|\boldsymbol{E}_{v}^{\text {in }}\right|^{2} N_{c} \epsilon^{2}$. The final accuracy of a calculation depends mainly on the number of iterations and on the resolution, i.e., the number of subcubes per wavelength in the material. To obtain an accurate result, the resolution must be such that the approximations mentioned above are justified. In practice, the necessary resolution can be determined by comparing computed results where possible with exact results obtained, for instance, using the Mie theory for spheres.

We have implemented the whole procedure in FORTRAN 77 on a Convex $\mathrm{C} 1$ minisuper computer. The required CPU time to solve equation (7) for a particular situation is given by $t=$ $c N_{i} N_{c}^{2}$, where $c$ is a constant and $N_{i}$ is the number of required iterations. For the Convex $\mathrm{C} 1, c \approx 4 \times 10^{-5} \mathrm{~s}$. The memory requirement is approximately 384 bytes per subcube using single precision arithmetic.

\section{RESULTS}

\section{a) Test Results}

We have tested our code in various ways. First, we have recalculated the electric field values tabulated by Livesay and Chen (1974) in their Figures 4 and 5 (to 4 and 3 significant figures, respectively), which they obtained by solving equation (7) numerically for the case of a finite slab illuminated by a plane wave. We used the same configuration of subvolumes as
Livesay and Chen, and we set the error criterion $\epsilon$ to $10^{-6}$. We found that our results agreed to one unit is the last decimal with the numbers in their Figure 4 . We could reproduce the numbers in their Figure 5 with a maximum deviation of 0.004 for the $x$-component of the field and 0.011 for the $z$ component. We do not know the cause for these small but significant discrepancies, but we note that Livesay and Chen do not specify their method of solving the matrix equation (12). Nevertheless, this test gives us confidence that we have made no major programming mistakes, as is confirmed by further comparisons.

Second, we have applied our code to approximate the scattering by a sphere $(m=1.7-0.1 i)$ and compared our results with numbers calculated with the coupled dipole method (by Draine 1988) as well as with the exact Mie theory. In our Figure 4 we reproduce part of Figure $3 d$ of Draine. We have taken from Draine the points showing the deviation between the precise Mie results for the extinction by spheres and his results using the coupled dipole method, obtained with an ensemble of 1064 dipoles. We replaced every dipole in this ensemble with a small cube, in which the electric field was assumed constant. The error criterion was set to $\epsilon=10^{-4}$. The results of this procedure are shown as plus signs in Figure 4. The number of iterations required ranged from $5\left(\mathrm{ka} / \mathrm{ka} a_{\mathrm{crit}}=\right.$ $0.15)$ to $40\left(k a / k a_{\text {crit }}=1.35\right)$. It can be seen that our method provides a fit to the precise Mie results that is comparable to or better than the coupled dipole method, for this particular case, and this appears to be due to the inclusion of a term proportional to $k^{2}$ in our formalism (see Appendix). For the cases we have tested, with $0 \leq X \leq 5$, our results differed from the Mie calculations by at most $4 \%$ for the extinction efficiency. The albedo and absorption efficiency differed by less than $5 \%$ from the Mie theory for the same range in $X$ using our method.

\section{b) Results for Porous Particles}

The symbols in Figures $5 a, 5 b, 6 a$, and $6 b$ show the results of calculations for the model porous aggregates described in $\S$ II. For these calculations we have set $\epsilon=10^{-4}$, and we used four

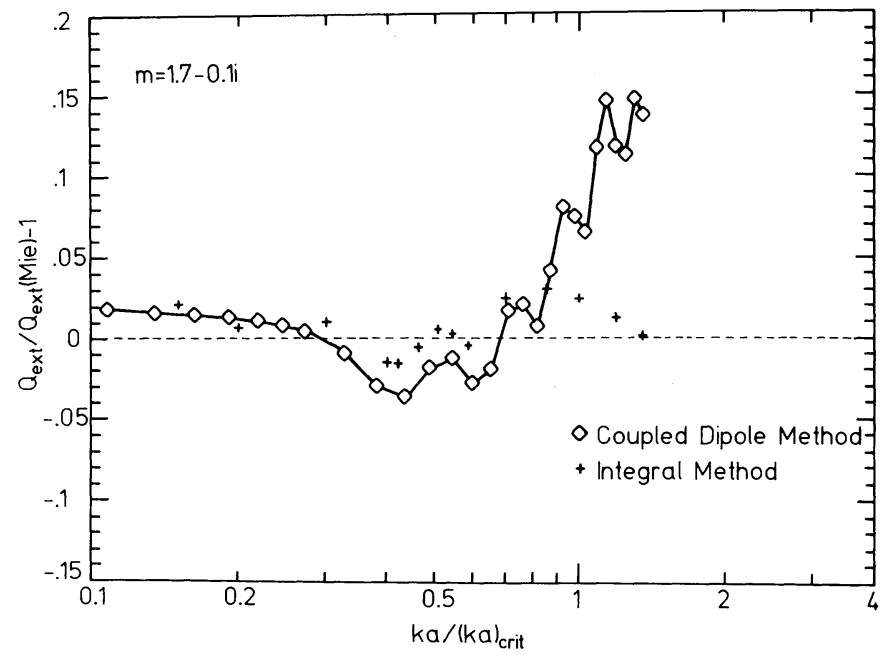

FIG. 4.-Deviations of the approximate values for $Q_{\mathrm{ext}}$ from the precise values obtained with Mie theory. Diamonds: values obtained using the coupled dipole method and an ensemble of 1064 dipoles. Plus signs: values obtained using eq. (7) and an ensemble of 1064 cubes. This figure, excluding the crosses, was adapted from Fig. $3 d$ of Draine $(1988)\left[(k a)_{c r i t}=3.70\right]$. Note that we use a negative imaginary part in the refractive index. 


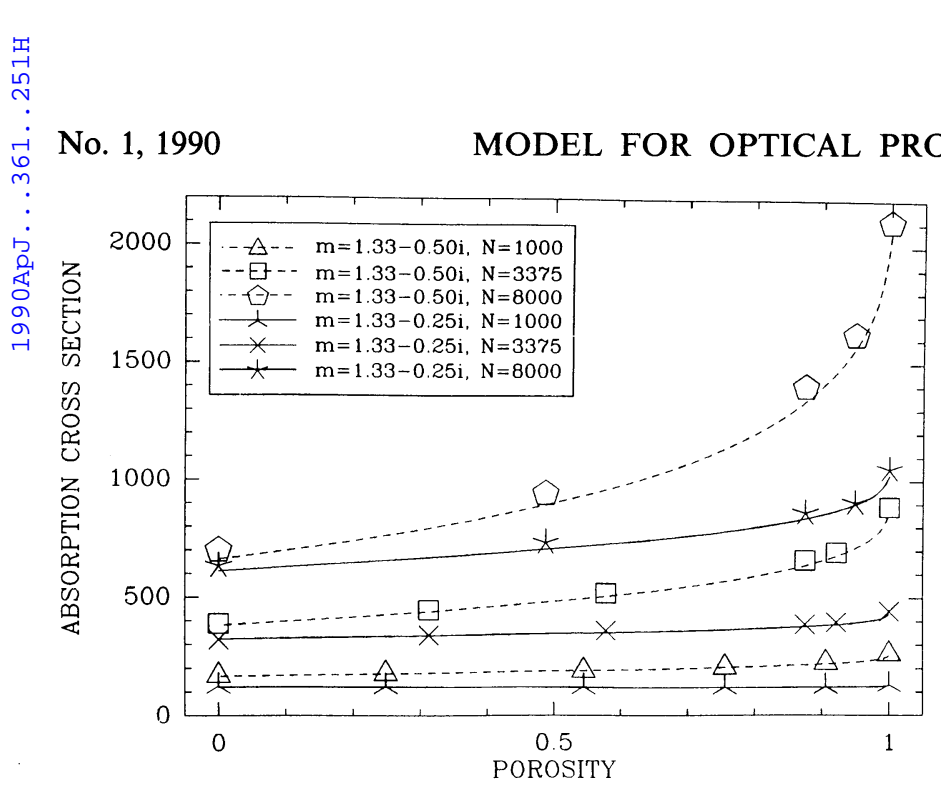

FIG. $5 a$

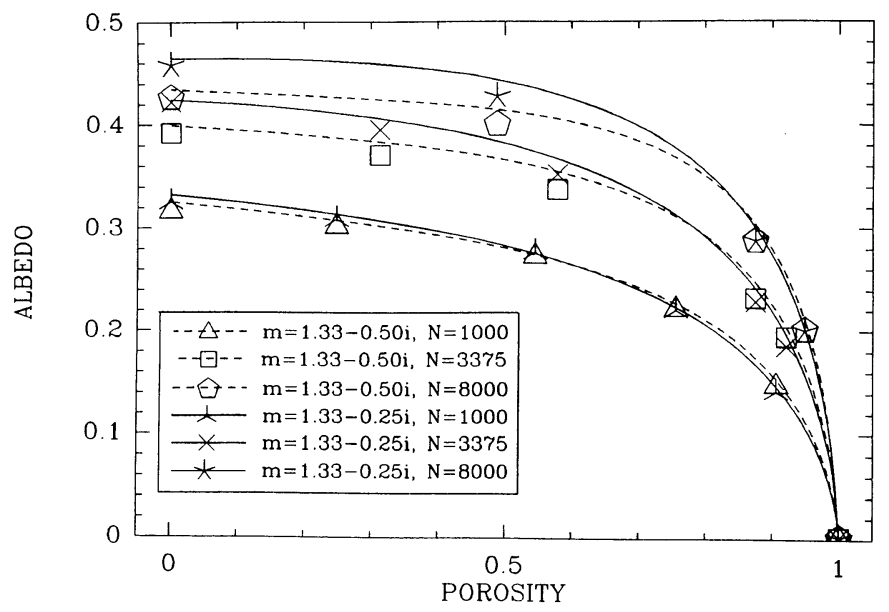

Fig. $5 b$

Fig. 5.- (a) Absorption cross section for various porous aggregates. Symbols show results obtained by solving eq. (7). The curves were calculated using Maxwell-Garnett effective medium theory in combination with Mie theory. The refractive index of the material is either $m=1.33-0.25 i$ or $m=1.33-0.5 i$. The number $N$ denotes how many subvolumes (which have a size parameter of 0.19 ) are contained in the aggregates. At $P=0, N=10^{3}, N=15^{3}$, and $N=20^{3}$ correspond to solid aggregates with size parameters equal to $1.9,2.8$, and 3.8 , respectively. The unit of area in which the absorption cross section is shown is equal to one face of a subvolume. $(b)$ Same as $(a)$ but showing the albedos of the aggregates.

different refractive indices. The number of iterations required ranged from 2 to 10 . Figures $5 a$ and $5 b$ pertain to the absorption cross sections and albedos, respectively, of aggregates containing subvolumes which all have $m=1.33-0.25 i$ or containing subvolumes all of which have $m=1.33-0.5 i$. Figures $6 a$ and $6 b$ show the results for aggregates which have inclusions with $m=1.7-0.25 i$ or inclusions with $m=1.7-0.5 i$. The area of one face of a subvolume is used as the unit area in Figures $5 a$ and $6 a$.

Consider the three-armed crosses closest to the lowest curve in Figure 5a. These crosses give the results of calculations for aggregates all of which are built up of 1000 cubical subvolumes (in this case with $m=1.33-0.25 i$ ) but which have different porosities. At $P=0$ these 1000 subvolumes, each with $x \approx 0.19$, are packed together in a $10 \times 10 \times 10$ cubic lattice, forming a solid cube with a size parameter $X \approx 1.9$. The points at $P>0$ represent aggregates containing 1000 subvolumes which are now randomly mounted in a cubic lattice that is larger than $10 \times 10 \times 10$. For example, an aggregate with porosity $P \approx 0.545$ is obtained by randomly distributing the 1000 cubical subvolumes on a cubic $13 \times 13 \times 13$ lattice. We have used a random number generator available on our computer to calculate the coordinates of the filled subvolumes in the lattice. Thus, increasing $P$ from 0 to 1 means going from a solid aggregate to a cloud of particles, while maintaining a constant amount of material.

The buildup of Figures $5 a$ and $6 a$ is as follows. The threearmed crosses and triangles closest to the bottom two curves in these figures correspond to aggregates containing 1000 subvolumes. The crosses and squares closest to the middle two curves in Figure $5 a$ and to the top pair of curves in Figure $6 a$ correspond to aggregates containing 3375 subvolumes. Finally,

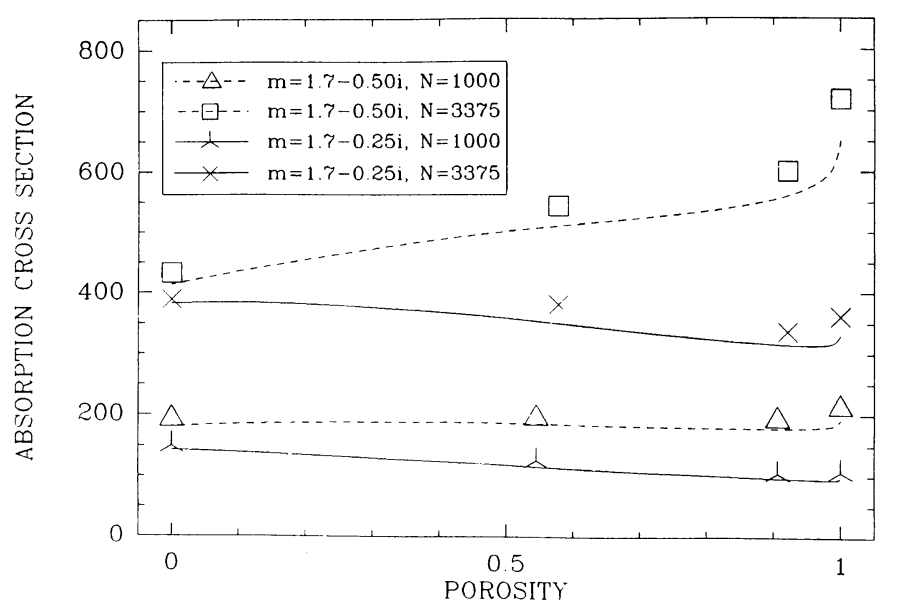

FIG. $6 a$

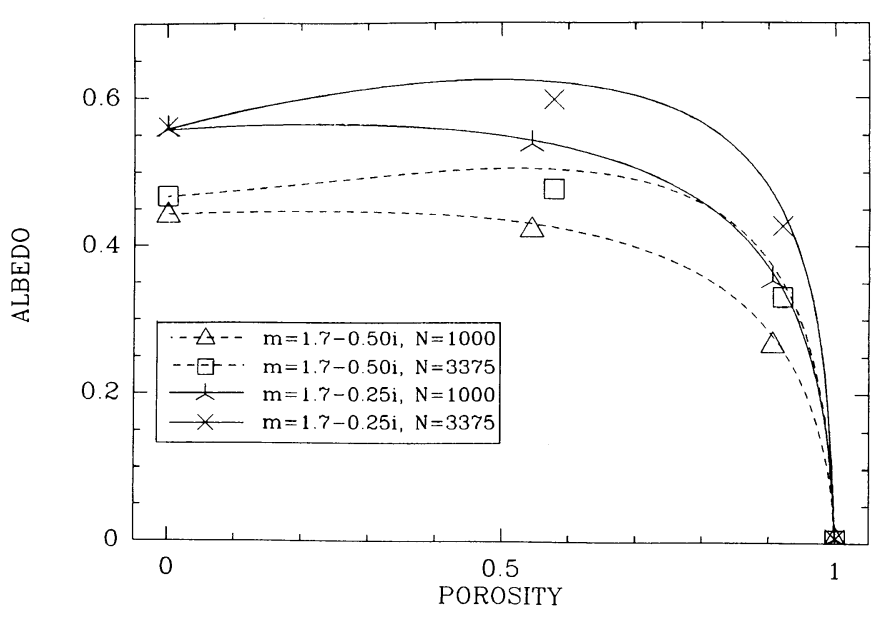

Fig. $6 b$

Fig. 6.-(a) Absorption cross section for various porous aggregates. Symbols show results obtained by solving eq. (7). The curves were calculated using Maxwell-Garnett effective medium theory in combination with Mie theory. The refractive index of the material is either $m=1.7-0.25 i$ or $m=1.7-0.5 i$. The number $N$ denotes how many subvolumes (which have a size parameter of 0.19 ) are contained in the aggregates. At $P=0, N=10^{3}$ and $N=15^{3}$ correspond to solid aggregates with size parameters equal to 1.9 and 2.8 , respectively. The unit of area in which the absorption cross section is shown is equal to one face of a subvolume. (b) Same as (a), but showing the albedos of the aggregates. 
the stars and pentagons close to the top pair of curves in Figure $5 a$ are the results of calculations made for aggregates of 8000 subvolumes. Figure $5 b$ applies to the same aggregates as Figure $5 a$, but shows their albedos. As in Figure $5 a$, the bottom, middle, and top groups of results correspond to aggregates containing 1000, 3375, and 8000 subvolumes. Figure $6 b$ shows the albedos of the aggregates used in Figure $6 a$. The ordering is as follows: crosses, 3375 subvolumes; three-armed crosses, 1000 subvolumes; squares, 3375 subvolumes; triangles, 1000 subvolumes.

The limiting values for $P=0$ and $P \approx 1$ can be calculated independently and used as a check. At $P=0$, the values of $C_{\text {abs }}$ and the albedo should be close, but not exactly equal, to those of spheres with an equal volume and the same refractive index. At porosity $P=1-\delta$, with $\delta \ll 1, C_{\text {abs }}$ should approach exactly the sum of the absorption cross sections of the individual subvolumes. The albedo at this extreme should equal the albedo of a single subvolume. The limits at $P=1$ are also shown.

Figures $5 a$ and $6 a$ illustrate the following points. Relatively compact particles in the considered size range may not show a change in absorption cross section (i.e., saturation has set in) when the imaginary part of the refractive index changes, i.e., if the material of which they are made becomes more, or less, absorbing. Compare, for example, the points for $m=1.33-0.25 i$ and $m=1.33-0.5 i$ in Figure $5 a$ at zero porosity. On the other hand, aggregates which contain the same amount of material but which are significantly porous show a change in absorption cross section nearly proportional to the change in $|\operatorname{Im} m|$, just like particles in the Rayleigh limit with a moderate index of refraction. Notice that a significant rise in absorption as a function of porosity only starts at a fairly high value of $P$. Furthermore, the degree of saturation depends on the size of the aggregates relative to the wavelength. This is illustrated by, e.g., the pairs of points for $m=1.33-0.25 i$ and $m=1.33-0.5 i$ which are relatively farther apart at a certain porosity for the smaller aggregates than for the larger aggregates for most of the range in $P$. Physically, the saturation of $C_{\text {abs }}$ for absorbing, relatively compact, and large particles is related to the fact that the incident wave does not penetrate completely into them, so that not all material inside can absorb energy. For a fixed refractive index and porosity, the larger the aggregate, the smaller the fraction of its volume which effectively absorbs energy. This volume fraction decreases in such a way that the absorption efficiency goes to a value close to unity as the size tends to infinity. All in all, Figures $5 a$ and $6 a$ show that a relatively large and solid particle, made of a material which has an intrinsic absorption profile, may not show a corresponding absorption or emission band, whereas a sufficiently porous aggregate containing an equal amount of the same material will. As far as the albedo of the aggregates is concerned, Figures $5 b$ and $6 b$ show that the major result of increasing the porosity of an absorbing aggregate, whilst keeping all other variables the same, is a decrease in the albedo. The decrease in albedo sets in at around $P \approx 0.75$ in these figures.

The curves shown in Figures $5 a-6 b$ were computed not by solving equation (7) but by representing a porous aggregate by a sphere equal in volume to the bounding volume of the aggregate and using an effective refractive index to calculate its optical properties. The scattering calculations for spheres were done using standard Mie theory. The effective refractive index was determined using Maxwell-Garnett's effective medium theory (Maxwell-Garnett 1904; Bohren and Huffman 1983), taking vacuum $(m=1)$ as the so-called matrix material and the filled subvolumes as the "inclusion." The effective refractive index, $m_{\mathrm{av}}$, is then given by equation (13):

$$
m_{\mathrm{av}}^{2}=1+\frac{3(1-P)\left(m^{2}-1\right) /\left(m^{2}+2\right)}{1-(1-P)\left(m^{2}-1\right) /\left(m^{2}+2\right)} .
$$

It can be shown that the absorption cross section computed in this way has the same limiting values at $P \approx 1$ and $P=0$ as given above, as long as the basic units in the aggregate are assumed to satisfy $x \ll 1$. In particular, at $P=0$, it follows from equation (13) that $m_{\mathrm{av}}=m$, and we have a solid sphere as above. In the limit $P \rightarrow 1-\delta=1-V_{\text {solid }} / V$ and $\delta \ll 1$, where $V$ is the bounding volume of the aggregate and $V_{\text {solid }}$ the volume of all the subparticles together, we have, using equation (13),

$$
m_{\mathrm{av}}^{2}=1+\frac{3 \delta\left(m^{2}-1\right) /\left(m^{2}+2\right)}{1-\delta\left(m^{2}-1\right) /\left(m^{2}+2\right)} \approx 1+3 \delta\left(\frac{\epsilon-1}{\epsilon+2}\right) .
$$

The absorption cross section of a spherical volume $V$ having a refractive index $m_{\mathrm{av}}$, with $m_{\mathrm{av}}^{2}-1 \ll 1$ (van de Hulst 1957) is, using $\delta=V_{\text {solid }} / V$,

$$
C_{\mathrm{abs}}=-k V \operatorname{Im}\left(m_{\mathrm{av}}^{2}-1\right)=-3 k V_{\text {solid }} \operatorname{Im}\left(\frac{m^{2}-1}{m^{2}+2}\right),
$$

and the absorption by a cloud of $N$ small spherical particles (which have nearly the same cross section as equal-volume cubes, as long as $x \ll 1$ ) with refractive index $m$ and radius $a$ which obey the condition $x \ll 1$ is (van de Hulst 1957)

$$
-3 N k\left(\frac{4 \pi}{3}\right) a^{3} \operatorname{Im}\left(\frac{m^{2}-1}{m^{2}+2}\right)=-3 k V_{\text {solid }} \operatorname{Im}\left(\frac{m^{2}-1}{m^{2}+2}\right),
$$

which is the same as equation (15). Figures $5 a$ to $6 b$ show that the agreement between the two approaches depends on the size parameter and refractive index, but is generally good. The maximum deviations between the curves and the symbols shown are $5 \%, 7 \%, 9 \%$, and $6 \%$ for Figures $5 a, 5 b, 6 a$, and $6 b$, respectively.

Because Mie theory combined with Maxwell-Garnett effective medium theory works so well for our model fluffy aggregates, we have extended our calculations for the fluffy particles, but now using equation (13) and Mie theory for spheres instead of solving equation (7) for each aggregate. The advantage is, of course, that Mie theory calculations require much less computer time than calculations using the integral equation (7). We show an application in Figures $7 a, 7 b$, and $7 c$. Each curve in these figures gives the thermal flux around $9.7 \mu \mathrm{m}$ emitted by one silicate aggregate with a certain porosity and a temperature of $420 \mathrm{~K}$. The fluxes are calculated using equation (1) and using the optical constants for (amorphous) "astronomical silicates" as given by Draine (1985). In a single figure all aggregates have the same amount of solid material but differ in their porosity. All the curves have their maximum normalized to unity.

It can be seen from Figures $7 a-7 c$ that the higher the porosity, the sharper the silicate emission becomes. At $P=0.99$, the emission for the cases shown is close in shape to the emission of a particle in the Rayleigh limit $(x \ll 1)$, which produces the narrowest feature possibile. At the other extreme, spheres with a radius of greater than, say, $5 \mu \mathrm{m}$ and with $P \approx 0$ hardly show an emission band at all, but emit like a blackbody, because the absorption has saturated. 


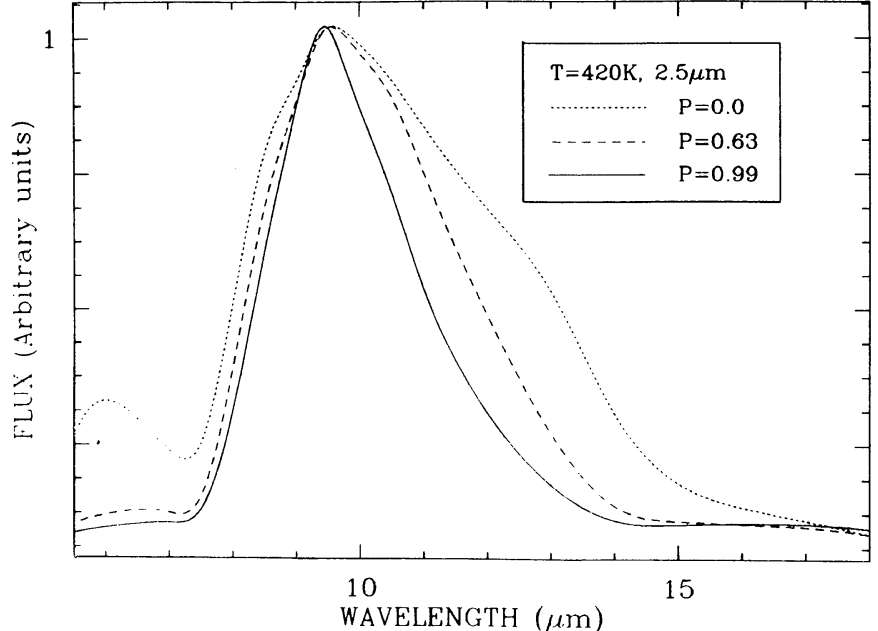

FIG. $7 a$

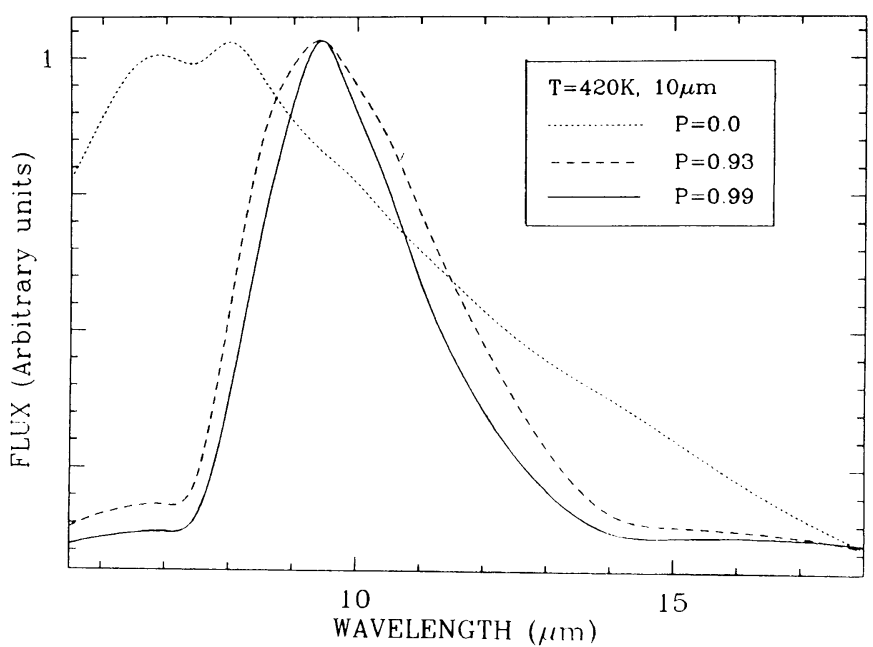

FIG. $7 c$

Fig. 7.- Thermal emission by equal-mass, porous silicate spheres at a temperature of $420 \mathrm{~K}$. (a) Porous spheres having the same amount of material as a solid sphere with a radius of $2.5 \mu \mathrm{m}$. (b) Same, for a radius of $5 \mu \mathrm{m}$. (c) Same, for a radius of $10 \mu \mathrm{m}$.

\section{DISCUSSION}

We can apply the curves in Figures $7 a-7 c$ to the emission observed from the coma of comet Halley as follows. The 9.7 $\mu \mathrm{m}$ silicate emission band of the comet as observed by Bregman et al. (1987) on 1985 December 12, has a full width at half-maximum (FWHM) of roughly $3.5 \mu \mathrm{m}$. The band is a composite of emission by amorphous and crystalline silicate (see Greenberg, Zhao, and Hage 1989 for a discussion of this), so that the FWHM of the emission by amorphous silicate alone is very probably less than $3.5 \mu \mathrm{m}$. Let us now suppose that the emitting particles can be represented by our model of fluffy aggregates, and let us use as a constraint that the FWHM of the emission is at most $3.5 \mu \mathrm{m}$. We can see from Figure $7 a$ that aggregates having the same volume of material as compact spherical particles with a radius of $2.5 \mu \mathrm{m}$ and $T=420 \mathrm{~K}$ must be at least $63 \%$ porous to fulfill the constraint. For larger aggregates, containing the same amount of material as solid spheres with radii of 5 and $10 \mu \mathrm{m}$, we find minimum porosities of $83 \%$ and $93 \%$, respectively. Particles

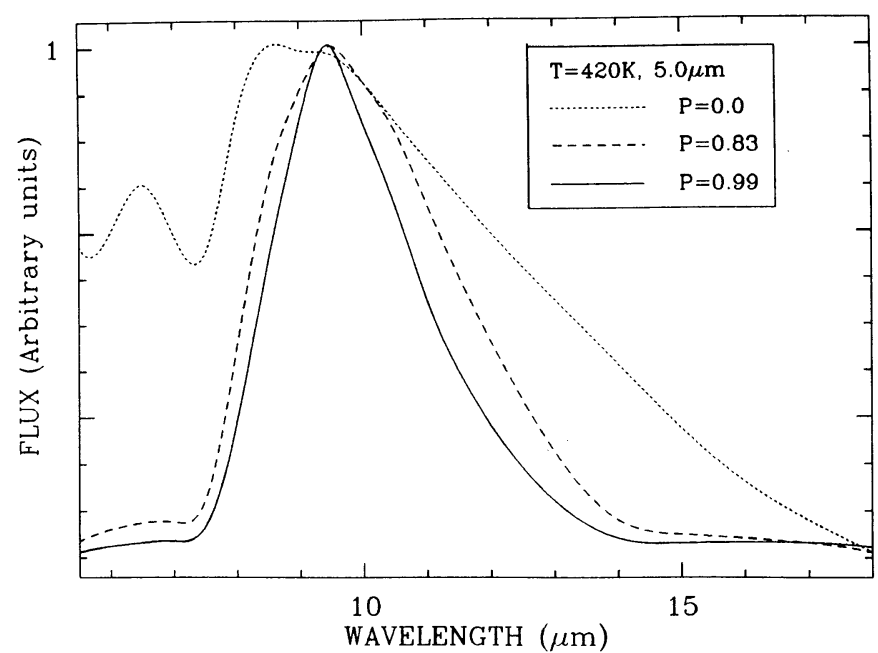

FIG. $7 b$

containing the same amount of material as a solid sphere with a radius of $\approx 1 \mu \mathrm{m}$ or smaller (not shown) need not be porous to emit an emission band with a FWHM narrower than 3.5 $\mu \mathrm{m}$. Decreasing the temperature to $320 \mathrm{~K}$ (the color temperature of the comet dust at the time of observation) raises the required minimum porosities to $82 \%, 93 \%$, and $97 \%$ because of the change in the Planck function. Adding an impurity to the aggregates, e.g., adding an absorbing carbon-rich coating on the pure silicate subparticles, may increase the required porosities if the extra material tends to depress the silicate emission. Therefore, if the emitting particles in the coma of comet Halley can be represented by the type of particles we have considered, then they must be either smaller than about 1 $\mu \mathrm{m}$ in radius or fluffy.

As a few extra checks on the model of porous aggregates, we have performed additional calculations for the particles used for Figures $5 a-6 b$, but now taking different polarizations and directions of the incident beam and also using different random placements of the subvolumes. The resulting differences in cross sections compared with those of Figures $5 a-6 b$ are small (less than about $5 \%$ in all cases checked). To check the validity of the combined Mie theory and Maxwell-Garnett approach in Figures $7 a-7 c$, we have calculated a few points on the curves by solving equation (7). The resulting difference in FWHM, calculated from equation (7) for a $90 \%$ porous aggregate, was $5 \%$.

The practical upper limit on the size range we have considered is constrained by the available amount of computer time, but we believe that we have considered a sufficient range in aggregate size to give some insight into the behavior of porous aggregates. The results presented in Figures $5 a-6 b$ also depend on the refractive index. We have chosen the real part of the refractive index equal to 1.33 and 1.7 because both of these values make calculations feasible, but also because 1.33 is a representative number often used in scattering calculations and 1.7 can be considered as representative of silicates at around $\lambda=9.7 \mu \mathrm{m}$. The typical features of absorbing aggregates we have found, namely, enhanced absorption and a lower albedo when the porosity is high, will still apply for other refractive indices to a certain degree, as long as the subparticles in the aggregate are not totally nonabsorbing or metallic. The properties of other porous aggregates can be calculated using the Maxwell-Garnett theory, provided that the subparticles in the aggregate are small compared to the wavelength. We 
expect that aggregates containing absorbing subparticles that are not small compared with the wavelength will also show enhanced absorption and a lower albedo, but in that case the approximation using Maxwell-Garnett theory will break down.

VII. CONCLUSIONS

Solving the integral equation (7) for our model of fluffy particles has proved to be a workable approach to calculate the scattering by porous aggregates. The method agrees well with the approach using Maxwell-Garnett effective medium theory in combination with Mie theory. With a few test cases we have indicated that the absorption cross section and the albedo of aggregates containing randomly placed inclusions with a small size parameter are well described using the latter approach. The extinction can also be computed to the same accuracy in this way. We conclude that the results show that vacuum has to be used as the matrix material in the Maxwell-Garnett theory, if scattering by fluffy aggregates is to be computed. Using vacuum as inclusions produces good results at $P \approx 0$, but gives the wrong limiting value as $\boldsymbol{P}$ goes to unity.

It is not to be expected that the Maxwell-Garnett theory also applies to porous aggregates containing inclusions that are not small compared with the wavelength. That problem could, in principle, be tackled by solving equation (7), but the approach is eventually limited by the fact that the amount of computer time required increases with the sixth power of the typical linear size of the scattering particle. Solving equation (7) using the method described in this paper is not limited to porous aggregates. An interesting application might be, for example, to calculate the optical properties of particles with a rough surface. It is also possible to calculate the polarizing properties of a variety of scattering particles using equation (7).

We have tested our method for a small number of cases by comparison with results of the Mie theory and the coupled dipole method for spheres. The accuracy of the present method was comparable to or better than the accuracy of the coupled dipole method in these cases, but further calculations must be done to establish reliably the relative accuracies of both methods.

We are indebted to P. M. van den Berg for essential theoretical support and J. van Kats of Convex Computer BV for programming advice. We thank H. C. van de Hulst, J. W. Hovenier, and M. S. de Groot for their helpful suggestions during the course of this work.

\section{APPENDIX}

In this appendix we briefly show how the basic equation used in the coupled dipole method can be derived from the integral equation (7). We approximate a scattering particle by an ensemble of identical, small cubical subvolumes and take the electric field to be homogeneous in each subvolume. If, instead, we take the subvolume containing $\boldsymbol{r}_{1}$ to be an equivalent volume sphere with radius $a$, the integrand for this subvolume can be calculated analytically (see, for example, Livesay and Chen 1974). The result is that equation (6) becomes

$$
\boldsymbol{E}\left(\boldsymbol{r}_{1}\right)=\boldsymbol{E}^{\mathrm{in}}\left(\boldsymbol{r}_{1}\right)-\frac{m^{2}\left(\boldsymbol{r}_{1}\right)-1}{3} \boldsymbol{E}\left(\boldsymbol{r}_{1}\right)+\frac{2}{3}\left[m^{2}\left(\boldsymbol{r}_{1}\right)-1\right][(i k a+1) \exp (-i k a)-1] \boldsymbol{E}\left(\boldsymbol{r}_{1}+\frac{k^{2}}{4 \pi} \sum_{i} \iiint_{V_{\mathrm{sub}}}\left[m^{2}\left(\boldsymbol{r}_{2}\right)-1\right] \boldsymbol{E}\left(\boldsymbol{r}_{2}\right) \mathbf{G}\left(\boldsymbol{r}_{1}, \boldsymbol{r}_{2}\right) d^{3} \boldsymbol{r}_{2} .\right.
$$

The summation is over all subvolumes which make up the particle, and the integrals must be evaluated for each subvolume. It may be assumed that $\mathbf{G}\left(r_{1}, r_{2}\right)$ is nonvarying inside each subvolume also, and $m$ is a constant inside the particle and equal to zero elsewhere. Then the integrals drop out of equation (A1), and what remains is that the electric field in a subvolume $j$ can be expressed as a summation over the electric field values in the other subvolumes, as follows:

$$
E_{j}\left\{1+\frac{m^{2}-1}{3}-\frac{2}{3}\left(m^{2}-1\right)[(i k a+1) \exp (-i k a)-1]\right\}=E_{j}^{\text {in }}+\frac{k^{2}}{4 \pi} \sum_{i}\left(m^{2}-1\right) E_{i} \mathbf{G}_{i j} \delta V,
$$

where the indices serve to number the quantities pertaining to the different subvolumes and $\delta V$ is the volume of a subvolume. Equation (A2) can be written in terms of the total dipole moment of the subvolumes, $p_{i}$, by using the relation $p_{i}=E_{i} \delta V\left(m^{2}-1\right) / 4 \pi$, with the following result:

$$
\boldsymbol{p}_{j}\left\{\frac{1}{\alpha}-\frac{2}{a^{3}}[(i k a+1) \exp (-i k a)-1]\right\}=E_{j}^{\mathrm{in}}+k^{2} \sum_{i} \boldsymbol{p}_{i} \mathbf{G}_{i j},
$$

where we have used $\delta V=(4 \pi / 3) a^{3}$. We note that $\alpha=\left[3\left(m^{2}-1\right) / 4 \pi\left(m^{2}+2\right)\right] \delta V$ is the same $\alpha$ used in the coupled dipole method, as defined by Purcell and Pennypacker (1973). Taking $k a \ll 1$, equation (A3) becomes

$$
p_{j}\left[1-\alpha\left(\frac{k^{2}}{a}-\frac{2}{3} i k^{3}\right)\right]=\frac{1}{\alpha}\left(E_{j}^{\mathrm{in}}+k^{2} \sum_{i} \boldsymbol{p}_{i} \mathbf{G}_{i j}\right) .
$$

If the terms proportional to $\alpha$ on the left-hand side are omitted, equation (A4) is the same as the basic expression used in the original coupled dipole method (disregarding differences in sign due to our choice of conventions; see § III). Equation (A4) is different from the basic equation used by Draine (1988), who added the radiation reaction to the original coupled dipole formulation, only through the term $\alpha k^{2} / a$ (disregarding differences in sign). By recomputing a few points in our Figure 4, excluding the term $\alpha k^{2} / a$ from the calculations, we found essentially the same deviation from the Mie theory as Draine. This difference in the formalism is therefore the reason that the method presented here reproduced the scattering by a sphere with $m=1.7-0.1 i$ more accurately at larger $k$ than the coupled dipole method as it was used by Draine. 


\section{REFERENCES}

Bohren, C. F., and Huffman, D. R. 1983, Absorption and Scattering of Light by Small Particles (New York: Wiley).

Bregman, J. D., Campins, H., Witteborn, F. C., Wooden, D. H., Rank, D. M., Allamandola, L. J. Cohen, M., and Tielens, A. G. G. M. 1987, Astr. Ap., 187, 616.

Brownlee, D. E. Tomandl, D. A., and Hodge, P. W. 1976, in IAU Colloquium 31, Interplanetary Dust and Zodiacal Light, ed. $\mathrm{H}$. Elsässer and $\mathrm{H}$. Fechtig (Lecture Notes in Physics, Vol. 48; Berlin: Springer-Verlag), p. 279.

Draine, B. T. 1985, Ap. J. Suppl., 57, 587. 1988, Ap. J., 333, 848.

Giese, R. H., Weiss, K., Zerull, R. H., and Ono, T. 1978, Astr. Ap., 65, 265.

Greenberg, J. M. 1960, J. Appl. Phys., 31, 82.

1980, in IAU Symposium 90, Solid Particles in the Solar System, ed. I. Halliday and B. A. McIntosh (Dordrecht: Reidel), p. 343.

. 1982, in Comets, ed. L. L. Wilkening (Tucson: University of Arizona Press), p. 131 . .1985, Phys. Scripta, T11, 14

. 1986, Nature, 321, 385.

Greenberg, J. M., and Hage, J. I. 1990, Ap. J., 361, 260.
Greenberg, J. M., Zhao., and Hage, J. I. 1989, Adv. Space Res., 9 (No. 3), 3

Harrington, R. F. 1968, Field Computation by Moment Methods (New York: Macmillan).

Jackson, J. D. 1975, Classical Electrod ynamics (2d ed.; New York: Wiley). Jones, A. P. 1988, M.N.R.A.S., 234, 209.

Livesay, D. E., and Chen, K. 1974, IEEE Trans., MTT22, 1273.

Maxwell-Garnett, J. C. 1904, Phil. Trans. R. Soc. London, A, 203, 385

Morse, P. M., and Feshbach, H. 1953, Methods of Theoretical Physics (New York: McGraw-Hill)

Peterson, A. F., and Mittra, R. 1985, J. Opt. Soc. Am. A, 2, 971

Press, H. P., Flannery, B. P., Teukolsky, S. A., and Vetterling, W. T. 1986, Numerical Recipes (New York: Cambridge University Press)

Purcell, E. M., and Pennypacker, C. R. 1973, Ap. J., 186, 705.

van den Berg, P. M. 1984, IEEE Trans., AP32, 1063.

van de Hulst, H. C. 1957, Light Scattering by Small Particles (New York: Wiley; reprinted 1981, New York: Dover).

Verniani, F. 1973, J. Geophys. Res., 78, 8429.

Wright, E. L. 1987, Ap. J., 320, 818

Yaghjian, A. D. 1980, in Proc. IEEE, 68, 248.

J. M. GreEnBERg and J. I. HAGE: Laboratory Astrophysics, University of Leiden, Postbus 9504, 2300 RA Leiden, The Netherlands 